\title{
Multiphysics Analysis of W7-X Control Coils
}

\author{
Jiawu Zhu ${ }^{\mathrm{a},{ }^{*}}$, Victor Bykov ${ }^{\mathrm{a}}$, Gunnar Ehrke ${ }^{\mathrm{a}}$, Boris Mendelevitch ${ }^{\mathrm{b}}$, Dirk Naujoks ${ }^{\mathrm{a}}$, Lutz Wegener ${ }^{\mathrm{a}}$, \\ Hans-Stephan Bosch ${ }^{\mathrm{a}}$ and W7-X team \\ ${ }^{a}$ Max-planck Institute for plasma physics, Greifswald, Germany \\ ${ }^{b}$ Max-planck Institute for plasma physics, Garching, Germany
}

\begin{abstract}
The quasi-symmetric fivefold modular Wendelstein 7-X (W7-X) stellarator consists of three groups of coil systems, i.e. superconducting magnet, trim coil and control coil systems. The control coil system contains ten identical 3D shaped control coils (CC) situated behind the baffle plates of corresponding divertor units, and is designated to rectify the error field and to sweep hot spots on the divertor target plates. The CC is wound from copper conductor with a square cross section of $16 \mathrm{~mm} \times 16 \mathrm{~mm}$ and a water cooling hollow of $\varnothing 8 \mathrm{~mm}$. The control coil system was installed in W7-X in 2015, and the integral commissioning has been done in parallel with the completion of W7-X. During the operation phase (OP 1.2a) with limited plasma heating energy, a leakage in one of the CC cooling water plug-in was found and dictates a detailed transient thermal analysis of $\mathrm{CC}$ to determine the allowable operation time without cooling water flow. The paper presents the transient thermal analysis and is followed by a detailed finite element mechanical analysis with the consideration of temperature gradient loads, dead weight and electromagnetic forces. Moreover, the transient thermal and mechanical performance of actively cooled CC to be intensively operated during steady state operation phase (OP 2) are also analyzed and evaluated with the same FE model.
\end{abstract}

Keywords: Wendelstein 7-X (W7-X), stellarator, control coil, thermal analysis, electromagnetic analysis, mechanical analysis.

\section{Introduction}

Following the successful first operation phase OP1.1 of the advanced modular stellarator Wendelstein 7-X (W7-X) with limiter plasma configurations, the second operation phases OP1.2a and OP1.2b with inertially cooled test divertor units (TDUs) are started in May of 2017 and April of 2018 respectively. The heating power of $8 \mathrm{MW}$ in $10 \mathrm{~s}$ is limited by the TDUs and 2 TDU scraper elements, which are installed before OP1.2b for testing [1]. The control coil system, installed in $\mathrm{W} 7-\mathrm{X}$ in 2015, is also under commissioning during the second operation phase. It consists of ten identical 3D shaped control coils (CC) which are situated behind the baffle plates of corresponding divertor unit and designated to rectify the error field and to sweep hot spots on the divertor target plates [2-3]. Each CC winding pack comprises 8 turns (single pancake) of copper conductors, which are with a square cross section of $16 \mathrm{~mm} \times 16 \mathrm{~mm}$ and a water cooling hollow of $\varnothing 8 \mathrm{~mm}$. Fig. 1 shows one of the CC resides in the divertor volume formed by graphite shielded baffle modules and divertor closures [4]. The CCs are fed with ten individual power suppliers and operated independently with a dc current and a superimposed ac current. The main parameters of one CC are listed in Table 1 [2].

The feed through for $\mathrm{CC}$ is inside a plug-in (see Fig. 1) and delivers cooling water and electrical current through two hollow conductors, it is connected with the CC conductor leads through well matched plugs and sealed with double Orings. The connections are accomplished during the installation of CCs in 2015, however, a leakage is found in one of the connections during OP1.2a,

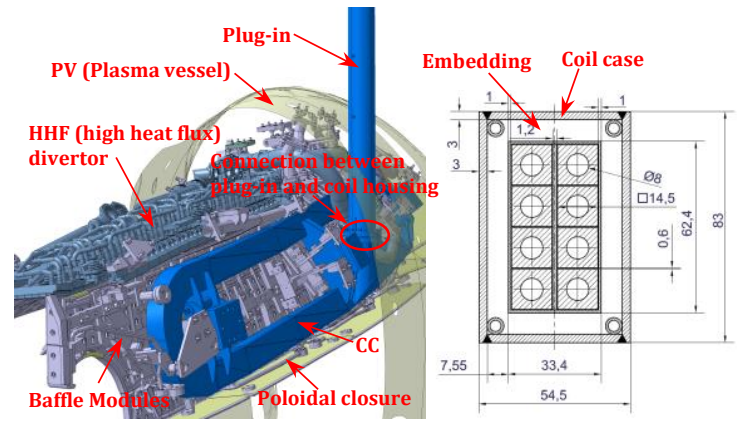

Fig. 1. View of one control coil situated inside of the divertor volume (part of PV is shown) and the coil section dimensions (unit: $\mathrm{mm}$ ). 
Table 1. Main parameters of one control coil

\begin{tabular}{ll}
\hline Designation & Value \\
\hline Inductance & $160 \mu \mathrm{H}$ \\
Resistance & $3.95 \mathrm{~m} \Omega$ \\
dc current & 0 to $\pm 2500 \mathrm{~A}$ \\
ac current & $625 \mathrm{Ap}$ \\
Max. eff. current & $2539 \mathrm{~A}$ \\
Frequency of the ac current & $0-20 \mathrm{~Hz}$ \\
Max. operation temperature & $60{ }^{\circ} \mathrm{C}$ \\
Max. temperature baking & $160{ }^{\circ} \mathrm{C}$ \\
\hline
\end{tabular}

which dictates a detailed transient thermal analysis of $\mathrm{CC}$ to determine the allowable operation time without cooling water. This paper at first studies the CC thermal behavior using a dedicated 3D FE model with the consideration of heat radiation, ohmic heating and ECRH (Electron-Cyclotron Resonance Heating), followed by the discussion and recommendation for $\mathrm{CC}$ operation. Secondly, the mechanical analysis with the consideration of temperature gradient loads, dead weight (DW) and Lorentz forces is presented and discussed. The Lorentz forces in CC are calculated using an electromagnetic (EM) model.

\section{Transient Thermal Analysis}

\subsection{FE Model}

As shown in Fig. 1, the control coil is surrounded by baffle modules (BM), TDU for OP1.2 or high heat flux (HHF) divertor for OP2, poloidal closure and plasma vessel (PV). The first wall elements are heated up during plasma operation [4]. As a result, radiant heat is concerned in CC thermal analysis, and realized by introducing a set of simplified FE model of the neighboring in-vessel components and PV (see Fig. 2).

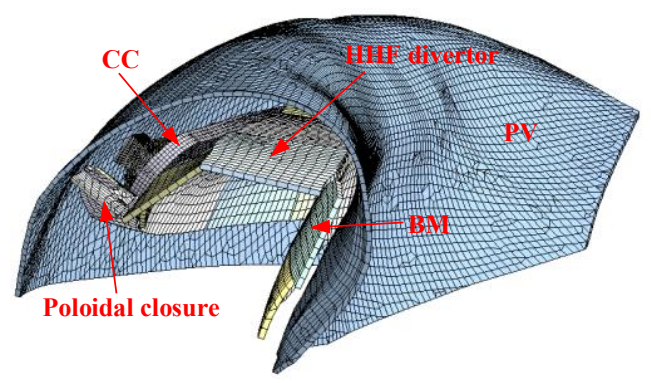

Fig. 2. FE model of simplified divertor volume for CC transient thermal analysis.

The CC inside the divertor volume is modeled with more details, as shown in Fig. 3, however the plug-in and feet through are excluded due to their negligible influences on thermal behavior. The 8 turns of conductor are modeled separately in simplified way, namely without the consideration of inter-turn and inter-pancake transitions. The most concerned CC winding pack (WP) is simulated with fine meshes with consideration of inter-turn, inter-layer and ground insulations. The insulations (glass fiber reinforced epoxy resin) are orthotropic materials which have different material properties in thickness direction and reinforcing plane specified in FE model by aligning the elements $\mathrm{x}$ coordinate direction with thickness direction (see Fig. 3c). The embedding fills the space between coil case and WP and is a mixture of ceramic sands $(\sim 50 \%)$ and epoxy resin. It is modeled as a isotropic material. Some bonded contacts are introduced to mimic the interfaces among WP, embedding, coil case and supports due to the inconsistence meshes. Four tubes at the corner of coil case (see Fig. 1) were temporary used for the epoxy resin impregnation, therefore they are excluded from FE modelling. The entire FE model comprises about 1.75 million nodes and 1.27 million elements (solid 90/87) generated in ANSYS Workbench $18.0^{\circledR}$.

\subsection{Scenarios for OP1.2}

During OP1.2, the TDU, BM and poloidal closure are operated in a passive way which limits the plasma heating power to $8 \mathrm{MW}$ at maximum, pulse length of $\sim 10 \mathrm{~s}$ and dwell time between two pulses less than $20 \mathrm{~min}$ [1]. The CC are to be also operated during the same plasma pulses to perform required physics program.

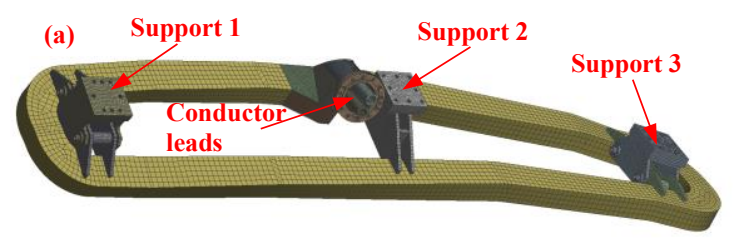



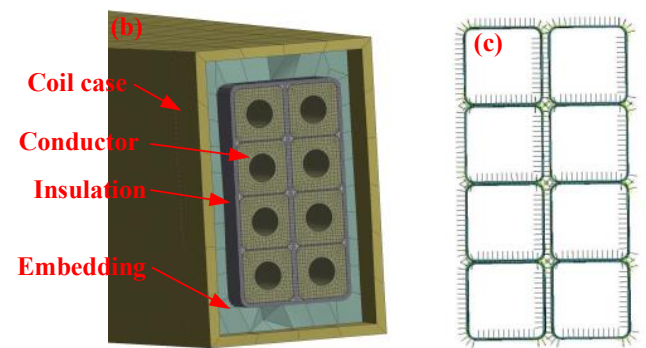

Fig. 3. FE model of 3D shaped control coil (a), mesh of coil section (b) and insulation elements orientation (c).

Consequently, the temperature of in-vessel components, as the boundary condition of thermal analysis, is also varied and substituted by the heat flux on plasma facing areas (for some analyzed cases) [5-7]:

- $\quad$ HHF divertor / TDU in high loaded area, $10 \mathrm{MW} / \mathrm{m}^{2}$,

- $\quad$ HHF divertor / TDU in low loaded area, $500 \mathrm{~kW} / \mathrm{m}^{2}$,

- $\quad$ Baffle modules, $250 \mathrm{~kW} / \mathrm{m}^{2}$,

- Poloidal closure, $100 \mathrm{~kW} / \mathrm{m}^{2}$.

The other parameters, such as emissivity, ECRH, heat generation, etc. of the analyzed scenarios are listed in Table 2. The temperatures of Case 1-3 are assumed for the scenario after 10 heating cycles according to the result of Case 1-1 and the calculation result reported in [6]. For conservative purpose, instead of 20 min the dwell time between two pulses is set to $10 \mathrm{~min}$. The $380 \mathrm{~W} / \mathrm{m}^{2}$ ECRH load absorbed by stainless steel (SS) surfaces is the result of $\sim 1 \%$ absorption rate of the ECRH power of $38 \mathrm{~kW} / \mathrm{m}^{2}$ in divertor volumes of Module 1 or 5 [7]. The cooling water convection coefficient for OP1.2 (Case 1-4) is about $15.7 \mathrm{~kW} / \mathrm{m}^{2} \cdot \mathrm{K}$ (calculated according to the operation parameters presented in [8]), and the bulk temperature of coolant is set to $50^{\circ} \mathrm{C}$. The criteria for CC safe operation from thermal point of view including:

- Temperature difference in WP less than $40{ }^{\circ} \mathrm{C}$ in order to avoid considerable thermal stress,

- Maximum temperature in WP less than $150{ }^{\circ} \mathrm{C}$ (the temperature of baking).

Table 3 collects the analysis results of all cases. Fig. 4 shows the WP temperature at the end of two heating cycles of Case 1-1. For OP1.2 with passive cooling, it is clear that the WP temperature could meet the operation requirements only in the case when the $\mathrm{CC}$ is operated starting from initial cold state and heated during not more than two cycles. In case of operation after 10 cycles with the same initial condition (Case 1-3), the WP maximum temperature reaches $150{ }^{\circ} \mathrm{C}$ in $1160 \mathrm{~s}$, and the temperature difference of $40^{\circ} \mathrm{C}$ in $255 \mathrm{~s}$. However, in case of water cooling (Case 1-4), the maximum temperature and temperature difference never reach the limits.

Table 2. Analysis scenarios and parameters of CC transient thermal analysis

\begin{tabular}{|c|c|c|c|c|c|c|c|}
\hline \multirow{2}{*}{\multicolumn{2}{|c|}{ Items }} & \multicolumn{4}{|c|}{ OP1.2 } & \multicolumn{2}{|c|}{ OP2 } \\
\hline & & ${ }^{\text {a } C a s e ~ 1-1 ~}$ & ${ }^{\mathrm{b}}$ Case $1-2$ & ${ }^{\circ}$ Case $1-3$ & ${ }^{\mathrm{d}}$ Case $1-4$ & ${ }^{\mathrm{e}}$ Case 2-1 & ${ }^{\mathrm{f}}$ Case 2-2 \\
\hline \multicolumn{2}{|c|}{ Time period, $\mathrm{s}$} & $\mathrm{g}_{604} \times 2$ & $604 \times 2$ & $604 \times 4$ & $604 \times 2$ & 250 & 1800 \\
\hline \multirow{7}{*}{$\begin{array}{c}\text { Temperature } \\
\left({ }^{\circ} \mathrm{C}\right) \text { and } \\
\text { emissivity }\end{array}$} & HHF TM5-6h &,-- 0.9 &,-- 0.9 & $280,0.9$ &,-- 0.9 & $399.4,0.2$ & $399.4,0.2$ \\
\hline & HHF TM7-9h &,-- 0.9 &,-- 0.9 & $900,0.9$ &,-- 0.9 & $145.5,0.47$ & $145.5,0.47$ \\
\hline & $\mathrm{BM}$ &,-- 0.2 &,-- 0.2 & $180,0.2$ &,-- 0.2 & $317.5,0.2$ & $317.5,0.2$ \\
\hline & Poloidal closure &,-- 0.5 &,-- 0.5 & $90,0.5$ &,-- 0.5 & $150,0.5$ & $150,0.5$ \\
\hline & PV & $60,0.5$ & $60,0.5$ & $60,0.5$ & $60,0.5$ & $60,0.5$ & $60,0.5$ \\
\hline & Ambient & 150 & 150 & 150 & 150 & 150 & 150 \\
\hline & Support & 60 & 60 & 60 & 60 & 60 & 60 \\
\hline \multicolumn{2}{|c|}{ ECRH, W/m $/ \mathrm{m}^{2}$} & $\mathrm{~h}_{380}$ & $\mathrm{~h}_{380}$ & $\mathrm{~h}_{3} 380$ & $\mathrm{~h}_{380}$ & 380 & 380 \\
\hline \multicolumn{2}{|c|}{ Conductor heat generation, $\mathrm{W} / \mathrm{m}^{3}$} & $\mathrm{~h} 4.428 \mathrm{e} 6$ & ${ }^{\mathrm{h}} 4.428 \mathrm{e} 6$ & $\mathrm{~h} 4.428 \mathrm{e} 6$ & h4.428e 6 & $4.428 \mathrm{e} 6$ & $4.428 \mathrm{e} 6$ \\
\hline
\end{tabular}

\footnotetext{
${ }^{\mathrm{a}}$ For OP1.2, passive cooling.

${ }^{b}$ For OP1.2, with water in cooling path but not circulating.

${ }^{\mathrm{c}}$ For OP1.2, assumed constant temperature of in-vessel component backside area after 10 heating cycles.

${ }^{\mathrm{d}}$ For OP1.2, active cooling, $\quad{ }^{\mathrm{e}}$ For OP2, passive cooling, $\quad{ }^{\mathrm{f}}$ For OP2, active cooling.

gTwo cycles, with $604 \mathrm{~s}$ per cycle, pulse length $10 \mathrm{~s}$ (including $2 \mathrm{~s}$ for heating power and current ramp up and down).

${ }^{\text {h}}$ Pulsed heating.
}

Table 3. Maximum temperature and temperature difference in CC WP

\begin{tabular}{lcccccc}
\hline Cases (see Table 2) & Case 1-1 & Case 1-2 & Case 1-3 & Case 1-4 & Case 2-1 & Case 2-2 \\
\hline Max. Temp. in WP during the solution time period, ${ }^{\circ} \mathrm{C}$ & 79.3 & 74.4 & 238.3 & 63 & 184.5 & 62 \\
Max. $\Delta$ Temp. in WP during the solution time period, ${ }^{\circ} \mathrm{C}$ & 40 & 36.4 & 156 & 18 & 110 & 29 \\
Time when Max. Temp. in WP reaching $150{ }^{\circ} \mathrm{C}$, s & $\sim 2420^{*}$ & $\sim 2720^{*}$ & 1160 & Never* & 175 & Never* \\
Time when Max. $\Delta$ Temp. reaching $40{ }^{\circ} \mathrm{C}$, s & 1208 & $\sim 1230^{*}$ & 255 & Never* & 47 & Never* \\
\hline
\end{tabular}

*Estimated according to the analysis result 


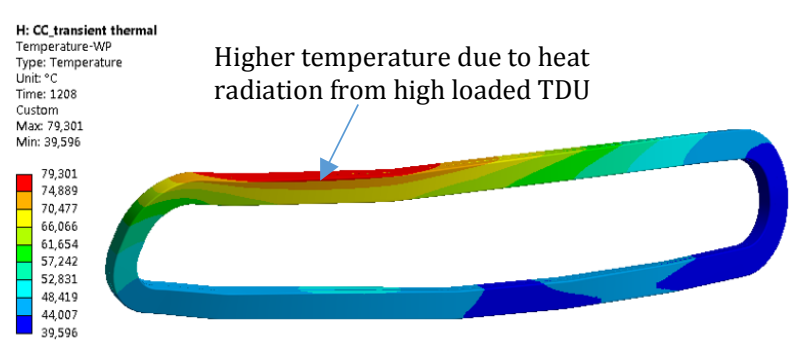

Fig. 4. Temperature distribution in WP at the end of two heating cycles of Case $1-1,{ }^{\circ} \mathrm{C}$.

\subsection{Scenarios for OP2}

The cooling water leakage issue is expected to be fully fixed before OP2, and the CC should be operated as designed in OP2. To verify the CC thermal performance during OP2, as listed in Table 2, two cases (passive and active cooling) are studied with the same FE model. The temperature and emissivity of in-vessel components backside area are set to the same values as the reference case of cryo-pump thermal analysis (with backside protection for BM and $25 \%$ shading effect from neighboring pipelines) [9]. The cooling water convection coefficient (for Case $2-2$ ) is about $280 \mathrm{~kW} / \mathrm{m}^{2} \cdot \mathrm{K}$ (calculated according to the operation parameters for OP2), and the bulk temperature of coolant is set to $50{ }^{\circ} \mathrm{C}$.

As listed in Table 3, in case of passive cooling (Case 2-1) and start of CC operation from initial cold state, the CC WP is heated up rapidly to $150{ }^{\circ} \mathrm{C}$ (max. allowed temperature) in less than $3 \mathrm{~min}$, and the state with temperature difference of $40{ }^{\circ} \mathrm{C}$ is reached in $47 \mathrm{~s}$. However, as expected, the maximum temperature and temperature difference are always meet the requirements in case of active cooling (Case 2-2). As a result, active cooling of CC is considered as mandatory for OP2.

\section{Mechanical Analysis}

\subsection{Electromagnetic forces}

The superconducting (SC) magnet system of W7-X consists of 50 non-planar coils of 5 types and 20 planar coils of 2 types. The coils are toroidally arranged in five equal modules. Due to the strong magnetic field generated by SC coils and interacted with the CC currents, the EM forces in CC conductors are non-negligible for mechanical analysis. To be conservative, one of the reference EM regime named 'low shear' (LS) with 3.0 T average magnetic induction on plasma axis is selected for CC EM force calculation, and the CC current is set to $2500 \mathrm{~A}$ with exclusion of ac current component. The force calculation is performed with coupled-field solid elements (solid 5) in ANSYS. The FE model of CC conductor is taken from the thermal analysis model. Due to the fact that external field is mainly in toroidal direction and almost parallel to the $\mathrm{CC}$ length direction, as shown in Fig. 6, EM forces in the CC middle part conductors are small, while the forces at the "ends" of CC are higher.

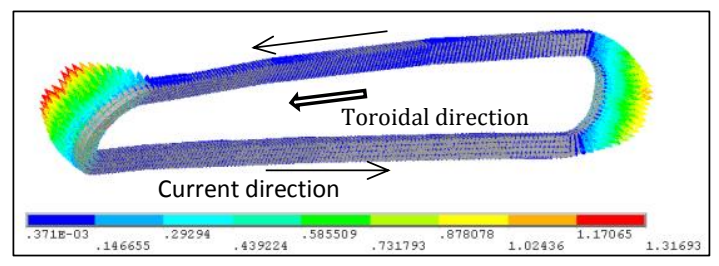

Fig. 6. EM forces in CC conductor elements, N.

\subsection{Mechanical analysis}

In addition to the EM forces, the DW and the temperature gradient from thermal analysis result of Case 1-1 and Case 2-2 are considered for mechanical analysis. The FE model (with element type solid186/187) is copied from thermal analysis with exception of the FE model parts of in-vessel components and PV, and with some modifications of contacts, i.e. the bearing contacts of support 1 and 3 are changed to 'frictional' (friction factor of 0.12) to allow CC thermal expansion. In order to obtain the primary stress, the mechanical solution is separated into two steps:

- $\quad$ Step 1 with DW and EM forces, which are regarded as primary loads;

- $\quad$ Step 2 with additional temperature loads to introduce the thermal stress (secondary stress).

Table 4. Mechanical assessment of coil case and conductor

\begin{tabular}{c|cccc}
\hline $\begin{array}{c}\text { Case } / \\
\text { Compone } \\
\text { nts }\end{array}$ & $\begin{array}{c}\mathrm{T}, \\
{ }^{\circ} \mathrm{C}\end{array}$ & $\begin{array}{c}{ }^{\mathrm{a}} \mathrm{m} / \text { limit, } \\
\mathrm{MPa}\end{array}$ & $\begin{array}{c}{ }^{\mathrm{b}} \mathrm{P}_{\mathrm{m}}+\mathrm{P}_{\mathrm{b}} / \\
\text { limit, } \mathrm{MPa}\end{array}$ & $\begin{array}{c}{ }^{\mathrm{c}} \mathrm{P}+\mathrm{Q} / \\
\text { limit, } \mathrm{MPa}\end{array}$ \\
\hline Case 1-1, & 50 & $22.7 / 36.7$ & $46.3 / 55$ & $73.5 / 110$ \\
conductor & 50 & $26.8 / 36.7$ & $31 / 55$ & $40 / 110$ \\
\hline Case 1-1, & 60 & ${ }^{*} 122 / 119$ & ${ }^{*} 180 / 178$ & $164 / 355$ \\
Coil case & 60 & ${ }^{*} 137 / 119$ & $154 / 178$ & $138 / 355$ \\
\hline
\end{tabular}


aPrimary membrane stress

bPrimary membrane plus bending stress

'Primary plus secondary stress

${ }^{*}$ Limits are low due to conservative yield stress from ITER

Table 5. Mechanical assessment of CC insulation

\begin{tabular}{lccc}
\hline \multicolumn{1}{c}{ Stress / strain } & Case 1-1 & Case 2-2 & Limits \\
\hline Max. Comp. stress, MPa & 142 & 115 & 800 \\
Max. Pri. tensile strain, \% & 0.17 & 0.17 & 0.0 \\
Max. Sec. tensile strain, \% & 0.095 & 0.15 & 0.02 \\
Max. in-plane strain, \% & $0.33 \sim$ & $0.31 \sim$ & \pm 0.5 \\
Max. shear stress, MPa & -0.12 & -0.11 & 56.7 \\
\hline
\end{tabular}

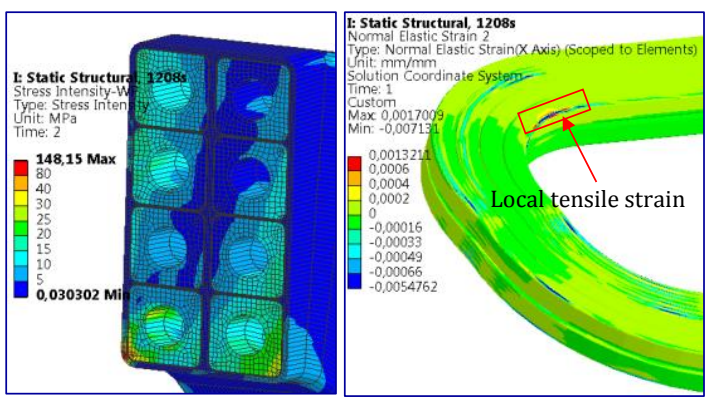

Fig. 7. Case 1-1, $\mathrm{P}+\mathrm{Q}$ stress at WP cross section (left) and primary tensile strain in turn insulation (right).

According to the design criteria for ITER in-vessel components [10], the stress / strain limits for metallic and nonmetallic structure assessment are defined and listed in Table 4 and 5 companying with the categorized stress / strain from the CC mechanical analysis. Two locations with high stress are identified for CC conductor / coil case assessment, as it is listed in Table 4. The primary loads for Case 1-1 and Case 2-2 are the same, but due to the temperature dependence of material properties, the $\mathrm{P}_{\mathrm{m}}$ and $\mathrm{P}_{\mathrm{m}}+\mathrm{P}_{\mathrm{b}}$ stresses are evaluated separately. Primary stresses slightly exceed the limits but only due to stress concentration, therefore, they are accepted. The assessment of CC insulation is listed in Table 5. Some tensile strains in thickness direction exceed the limits, however, the criteria in the project are to accept such local delamination for coil insulation as it was done also for the trim coils. It is well visible in Fig. 7 that the maximum normal tensile strain in turn insulation (the issue of most concern) is really local, and the normal tensile strains are mostly lower than $0.02 \%$, therefore, the insulation mechanical behavior is also acceptable.

\section{Conclusion}

Transient thermal behavior of possible operation scenarios for control coils of W7-X is studied with a dedicated 3D FE model, which is capable to consider the heat radiation loads, ohmic heating and ECRH. According to the analysis results of the $\mathrm{CC}$ with active cooling, the $\mathrm{CC}$ thermal performance is excellent for both OP1.2 and OP2. However, in case of passive cooling, it is required to operate the CC only starting from cold state with not more than two heating cycles of 20 min during OP1.2. The operation without cooling during OP2 is not possible. Mechanical analysis of CC with the considerations of EM forces, dead weight and temperature gradient indicates that the mechanical performance of both metallic structure parts and non-metallic structure elements (insulation) of CC are acceptable.

\section{Acknowledgment}

This work has been carried out within the framework of the EUROfusion Consortium and has received funding from the Euratom research and training programme 2014-2018 under grant agreement No 633053. The views and opinions expressed herein do not necessarily reflect those of the European Commission.

\section{References}

[1] H.-S. Bosch, T. Andreeva, et al., Engineering Challenges in W7-X: Lessons Learned and Status for the Second Operation Phase, IEEE Transactions on plasma science 46 (2018) 1131-1140.

[2] A. Peacock, The W7-X Control Coils and their Fabrication, W7-X project document, PLM: 1-ACG-Y0001.0.

[3] F. Füllenbach, T. Rummel et al., Final test of the W7-X control coils power supply and its integration into the overall control environment, Fusion Eng. Des. 83 (2007) 1391-1395.

[4] A. Peacock, J. Boscary, et al., Wendelstein 7-X High Heat Flux Components, IEEE $25^{\text {th }}$ Symposium on Fusion Engineering, DOI: 10.1109/SOFE.2013.6635360. 
[5] M. Ye, 3D FE thermal \& mechanical analysis of TDU, W7-X project document, PLM: 1-GXA50M-T0000.0

[6] M. Ye, Thermal analysis of passive divertor target and in-vessel components, W7-X project document, PLM: 1-GXA-T0140.0.

[7] R. Brakel, M. Köppen, A. Peacock, et al., Specification of Design Loads for in-vessel components of W7-X, W7-X project document, PLM: 1-AC-S0005.1.

[8] M. Braun, Cooling configuration for control coil, W7-X project document, PLM: 1-ACK30-Z0002.4.

[9] J. Zhu, V. Bykov, et al., Refined multiphysics analysis of W7-X cryopumps, IEEE Transactions on plasma science 46 (2018) 15921602.

[10] ITER Structural design criteria for in-vessel components (SDC-IC), IDM: 222RHC, ITER, Cadarache, France. 